\title{
Knowledge and management practices regarding dengue fever among doctors of tertiary care hospital
}

\begin{abstract}
Introduction: Worldwide incessantly menace "Dengue Fever" is a health phenomenon nowadays. We targeted three big provinces of Pakistan including Sindh, Punjab, and Khyber Pukhtoon Khwan. It makes a figure of 21,580 dengue cases and out of them, 317 patients fall in death group.

Materials and methods: A cross-sectional case-control study has been conducted at the general tertiary care hospital in Karachi, Pakistan. Physicians, Residents and Interns from all the specialty of targeted the hospital, were included. Four major sections were plotted i.e. general and specified knowledge of Dengue, Dengue symptoms knowledge, Knowledge of practices and management practices of Dengue fever.

Results: A randomized sample of 378 respondents was collected during the time period. In General, from 80 to $97 \%$ physician mark the correct answer of Dengue Fever Knowledge. It was strongly commented by the results that physicians have advance knowledge of Dengue symptoms. It was also observed that almost every physician has the knowledge Aspirin and Non-Steroidal Anti-Inflammatory Drugs must be avoided during the dengue fever or shock syndrome.

Conclusion: In spite of a major number of dengue patients in hit list cities, physicians have lack of knowledge about certain topics. Advance knowledge about dengue, symptomatic illness like pain behind the back of eyes is a symptom of dengue, vaccines of dengue, practices, and medication which should not be given to dengue fever or shock patients were found to be Do Not Know by the selected hospital physicians.
\end{abstract}

Volume 2 Issue 5 - 2018

\author{
Fahad Khan, Sham Kumar, Tayyab Raza Fraz, \\ Syeda Naqvi, Danish Kumar, Mashmula \\ Tahoor, Sanjay Kumar, Afia Rashid, Nadeem \\ Chandio \\ Internal Medicine, Jinnah Sindh Medical University, Pakistan
}

\begin{abstract}
Correspondence: Syeda Naqvi, Internal Medicine, Jinnah Sindh Medical University, Jinnah Postgraduate Medical Centre, Pakistan, Email syedafati240@gmail.com
\end{abstract}

Received: April 27, 2018 | Published: October 04, 2018

\section{Introduction}

Dengue fever has been an incessant menace worldwide. Approximately 2.5 billion people were explicated under the risk of suffering from dengue, especially those 975 million population living in urban and tropical countries during 2009. ${ }^{1,2}$ Dengue virus belongs to the family of Flaviviridae. It carries single-stranded RNA which causes arboviral disease. WHO claims a proportion of 4 out of 5 people are under the risk of parasitic transmission either by mosquitoes, ticks, fleas, flies or other. ${ }^{3}$ Although Dengue fever is radically preventable yet in some cases it causes shock syndrome and hemorrhagic fever which may lead to death. ${ }^{4}$ Researchers in the past reveals four types of dengue serotypes which have been circulating specifically during the post-monsoon period i.e. October and November epidemically in America, Africa and Asia. ${ }^{5-8}$ In Pakistan, the three provinces including Sindh, Punjab, and KPK has an incidence of 21,580 dengue cases, and among them, 317 patients died. ${ }^{9}$ In the light of previous research in Southern Taiwan, Central Nepal, North India, Sri Lanka, Malaysia, Jamaica, Singapore objective of this study was drawn. Moreover, research had been conducted in major cities of Pakistan on knowledge, attitude, practices, and management among physicians. ${ }^{10}$ That helped to evaluate dengue practices in terms of knowledge and management among physicians at tertiary care hospital of a major city of Pakistan i.e. Karachi.

\section{Materials \& methods}

A cross-sectional case-control study has been conducted at the general tertiary care hospital in Karachi, Pakistan from September 2015 to March 2016. A questionnaire has been designed on the basis of previous research conducted by Rafique et al published in JPMA $2015 .{ }^{10}$ After certain modification that covers all the leading questions with reference to knowledge, practices and management of all dengue patients were included further in the questionnaire. During the time period of data collection, Physicians and Resident from all the specialties were included. All doctors willing to be a part of this study and given consent were considered in the study population. Confidentiality was maintained by keeping all details anonymous. Moreover, a code was given to every questionnaire which only is decoded by authorized persons. With respect to the respondent, demographic sections were classified into a highest educational degree, designation, and posted ward additional with age, gender and marital status of the respondent. It was plotted into four sections i.e. General and specified knowledge of Dengue, Dengue symptoms awareness, knowledge of practices and management practices of Dengue fever. Descriptive analysis (frequency and percentage) were done in order to see the knowledge and practices gap among physicians. SPSS for window version of 21.0 was used for data analysis. For designing of a questionnaire, plotting the graph and figures we used Microsoft Excel.

\section{Results}

A randomized sample of 378 respondents was collected during the time period. Out of 378 respondents, the highest rewarding degree of respondent was observed to be fresh graduates $(n=369 ; 97.6 \%)$, 
while the ratio of Postgraduate trainees falls minimal $(\mathrm{n}=08,01 ; 2.1 \%$, $0.3 \%)$. These respondents were mostly female $(\mathrm{n}=250 / 378 ; 66.1 \%)$, however, both male and female were designated mostly in public hospitals $(93.9 \%)$. Most of the respondent who was willing to be a part of the study were under the age of 30 years $(n=350 ; 92.6 \%$ ) (Table 1$)$. For evaluating the knowledge about dengue fever in respect to general and specified, Section one: Knowledge of Dengue Fever; General and Advance comprises of eleven and five questions respectively. Each question had dichotomous variable i.e. Yes and No. In General, from $80 \%$ to $97 \%$ physician mark the correct answer of Dengue fever is caused by the mosquito; Aedes aegypti (Yes; 91.8\%), Stagnant water is the main source for mosquito breeding (Yes; 85.7\%), Dengue fever affects all age groups (Yes; 96.6\%), Control of dengue is by combating the breeding of mosquitoes (Yes; $90.2 \%$ ) and There is no specific treatment of dengue (Yes; 85.7). On the other hand, lack of knowledge was seen among the physicians in regrade of; Life cycle of the Aedes mosquito is one week (Yes; 37.0\%), Dengue epidemics start during hot weather (No; 36.2\%) and Dengue can be transmitted by direct blood contact (Yes; 25.9\%). In Advance, a serious lack of knowledge was observed i.e. physicians had blind knowledge about; There is a vaccine for Dengue (Yes; $11.9 \%$ ), Paracetamol [Panadol] is the drug of choice for dengue treatment (No; 38.1\%), Fatality Rate of patients diagnosed as Dengue Shock Syndrome (10\% Fatality Rate; $28.0 \%$ ), Discharge timing of the patients having stable Haematocrit levels, increased Platelet count and no Fever (48 Hours; 44.4\%) and
Criteria for Dengue Haemorrhagic Fever i.e. History of Fever lasting two to seven days, Haemorrhagic Manifestation, Thrombocytopenia and increased Vascular Permeability (All of the above; 44.4\%) See Table 2 .

Table I Demographic descriptive statistics of respondents

\begin{tabular}{llll}
\hline Characteristics & Groups & Frequency (N) & Percent (\%) \\
\hline Gender & Male & 128 & 33.9 \\
& Female & 250 & 66.1 \\
Age & $21-30$ Years & 350 & 92.6 \\
& $31-40$ Years & 15 & 4 \\
& 4I-50 Years & 4 & 1 \\
Qualification & $>50$ Years & 9 & 2.4 \\
& MBBS & 369 & 97.6 \\
& FCPS & 8 & 2.1 \\
& MRCP & 1 & 0.3 \\
Type of practice & Public & 355 & 93.9 \\
& Both (Public & 23 & 6.1 \\
\hline
\end{tabular}

Table 2 Descriptive statistics of section I- knowledge of dengue fever (general \& advance)

\begin{tabular}{|c|c|c|c|}
\hline S. No & General & Frequency $(n / 378)$ & Percentage (\%) \\
\hline I & Dengue fever is caused by the mosquito;Aedes aegypti (Yes) & 347 & 91.8 \\
\hline 2 & The life cycle of the Aedes mosquito is one week. (Yes) & 140 & 37 \\
\hline 3 & Stagnant water is the main source for mosquito breeding. (Yes) & 324 & 85.7 \\
\hline 4 & Dengue fever affects all age groups. (Yes) & 365 & 96.6 \\
\hline 5 & Dengue epidemics start during hot weather. (No) & 137 & 36.2 \\
\hline 6 & Dengue is a flu-like illness. (Yes) & 237 & 62.7 \\
\hline 7 & Dengue can be transmitted by direct blood contact. (Yes) & 98 & 25.9 \\
\hline 8 & Transmission cycle is "Man-Mosquito-Man". (Yes) & 267 & 70.7 \\
\hline 9 & Mosquitoes transmitting dengue infection bites only early in the morning. (No) & 229 & 60.6 \\
\hline 10 & Control of dengue is by combating the breeding of mosquitoes. (Yes) & 341 & 90.2 \\
\hline \multirow[t]{2}{*}{11} & There is no specific treatment for dengue. (Yes) & 324 & 85.7 \\
\hline & ADVANCE & & \\
\hline 12 & There is a vaccine for dengue. (Yes) & 45 & 11.9 \\
\hline 13 & Paracetamol $($ Panadol $\circledast)$ is the drug of choice for dengue treatment $(\mathrm{No})$ & 144 & 38.1 \\
\hline 14 & Fatality Rate of patients diagnosed with Dengue Shock Syndrome (10\% Fatality Rate) & 106 & 28 \\
\hline 15 & $\begin{array}{l}\text { Discharge timing of the patients having stable Hematocrit levels increased Platelet count and no } \\
\text { Fever ( } 48 \text { Hours) }\end{array}$ & 168 & 44.4 \\
\hline 16 & $\begin{array}{l}\text { Criteria for Dengue Hemorrhagic Fever i.e. History of Fever lasting 2-7 } \\
\text { days, Hemorrhagic Manifestation, Thrombocytopenia and increased Vascular Permeability (All of the } \\
\text { above) }\end{array}$ & 168 & 44.4 \\
\hline
\end{tabular}

Section two was designed to evaluate the Symptomatic Knowledge of Dengue Fever among the physicians. Total seven questions were designed to fill in order of Yes, No or Do Not Know statement. All the consecutive questions withhold the power of Yes; therefore, the ratio of the result was predicted in Percentage. It was strongly commented by the results that physicians have advance knowledge of Dengue Symptoms. It was also observed that the most controversial answer was the Abdominal Pain as a symptom of Dengue Fever beside the rest of six questions (Yes; $56.9 \%$, No; $38.4 \%$ \& Do not know; $4.8 \%)$. On the other hand, the majority of physicians were unaware 
of Pain behind the eyes is actually a symptom of Dengue Fever (Do Not Know; 28\%) Refer to Figure 1. A section (Section three: Dengue Medication) was also substituted on medications practices for dengue fever. Dengue activates hemorrhagic shock, therefore knowledge about medical practices regarding "Which medication should be avoided in case of hemorrhagic risk?" For this content, two options were used for evaluating correct or wrong practices among physicians. 358 out of 378 physicians $(94.7 \%)$ marked the correct practices which should be avoided in dengue fever i.e. Aspirin and Non-Steroidal Anti-Inflammatory Drugs. With references to the section, Excess use of among Crystalloids or Colloids or Ringer's Lactate during the stay at the hospital of dengue fever patients might cause Hyperchloremic Acidosis were inquired. Percentage of this answer was shocking, only $34.9 \%(n=132 / 378)$ physicians have knowledge that Crystalloids excessive use results in Hyperchloremic Acidosis in dengue fever patients during the hospitalization. In contrast, Ringer's Lactate which should be avoided in those dengue fever patients were asked to every respondent in this section as well. The options were designed for Patients having Liver disease or Diabetes or taking Metformin or Both Liver disease and taking Metformin; likewise, the previous question, lack of knowledge were observed among physicians i.e. Correct answer (Both Liver Disease and taking Metformin) picked out by $133(35.2 \%)$ physicians while 245 (64.8\%) marked wrong. For Assessing the Management Practices of Dengue Fever (During, Pre, and Post)-Section four, comprises of seven questions, which must be filled by every respondent. Each answer will be evaluated on practices by Always, Sometime and Never form. Majority of the respondent population practicing the management always see Figure 2. Besides, Tourniquet Test to Diagnose Dengue Infection and Performing of PCR in CSF for Dengue Meningitis were Never practiced by the physicians. On the basis of the analysis, the results are extracted into two forms i.e. positive results declared strong knowledge and practices in pre, post and during the dengue fever, while negative results reflect the blind knowledge and practices among physicians.
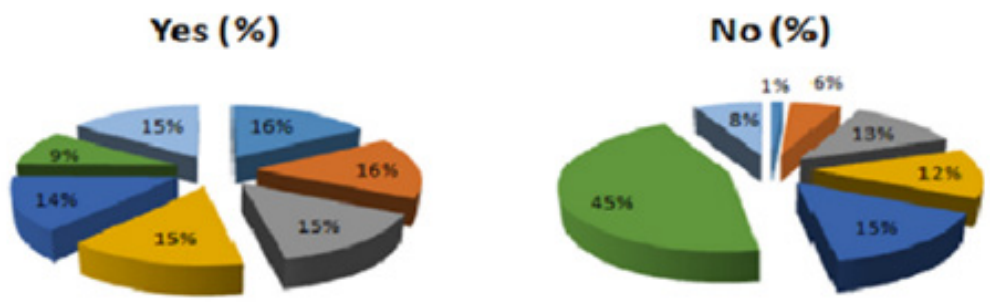

Do Not Know (\%)
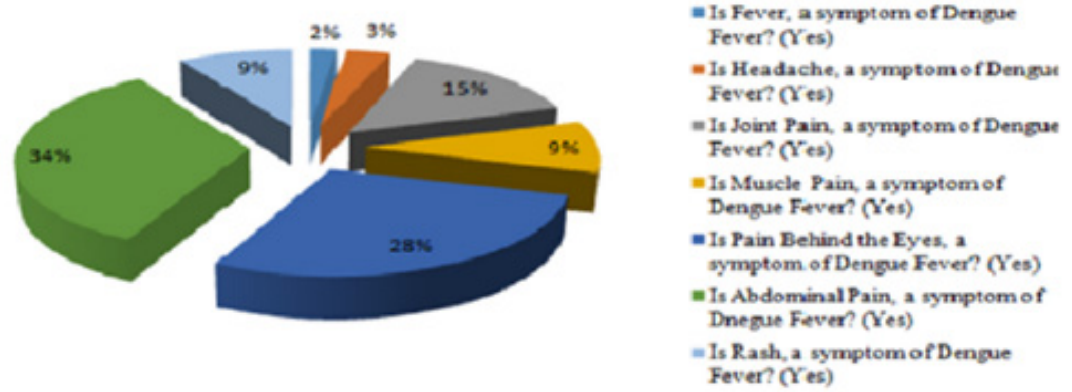

Figure I Symptomatic knowledge of dengue fever among the physicians. Each pie reflects the ratio/frequency of answers out of 378 .

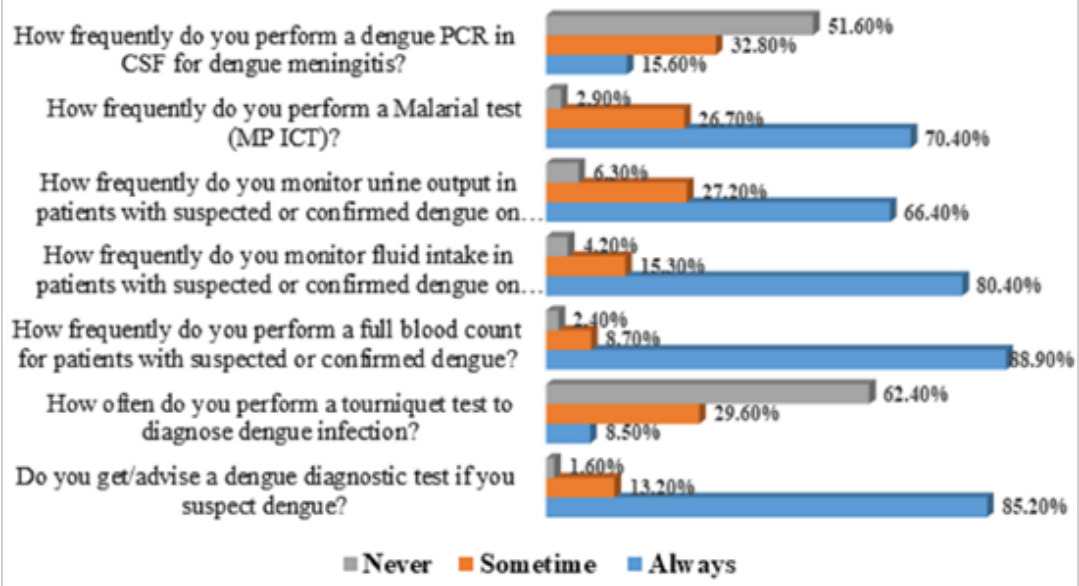

Figure 2 Descriptive statistics of dengue management follow-up by physicians. 


\section{Positive results}

\section{Section one: knowledge of dengue fever; general and advance}

In General, from 80 to $97 \%$ physician mark the correct answer of Dengue Fever Knowledge i.e. Dengue fever is caused by the mosquito; Aedes aegypti, stagnant water is the main source for mosquito breeding, Dengue fever affects all age groups, control of dengue is by combating the breeding of mosquitoes and there is no specific treatment of dengue.

\section{Section two: symptomatic knowledge of dengue fever}

All the consecutive questions withhold the power of Yes; therefore, the ratio of the result was predicted in Percentage. It was strongly commented by the results that physicians have advance knowledge of Dengue Symptoms.

\section{Section three: dengue medication}

It was observed that almost every physician had the knowledge Aspirin and Non-Steroidal Anti-Inflammatory Drugs must be avoided during the dengue fever or shock syndrome.

Section four: Management Practices of Dengue Fever (During, Pre, and Post)

On the basis of evaluation, practices which were being practiced by the physicians were marked as always practiced among the general tertiary care hospitals physicians.

\section{Negative results}

\section{Section one: knowledge of dengue fever; general and advance}

Lack of knowledge was seen among the physicians in regrading of; Life cycle of the Aedes mosquito is one week, Dengue epidemics start during hot weather and Dengue can be transmitted by direct blood contact. In Advance, a serious lack of knowledge was observed i.e. physicians had blind knowledge about specialized knowledge of dengue fever which a physician must have.

\section{Section two: symptomatic knowledge of dengue fever}

Likewise, the section one, lack of knowledge were also observed among the physicians about Abdominal Pain as a symptom of Dengue Fever and Pain behind the eyes is actually a symptom of Dengue Fever.

\section{Section three: dengue medication}

In this section physicians have blind knowledge about the practices that Crystalloids excessive use result in hyperchloremic acidosis in patients has dengue fever during the hospitalization. Furthermore, Ringer's Lactate which should be avoided in those dengue fever patients having both Liver and Metformin Disease were known by few physicians during the data collection time period.

Section four: management practices of dengue fever (during, pre, and post)

Out of seven questions, only one question i.e. Tourniquet Test to Diagnose Dengue Infection and Performing of PCR in CSF for Dengue Meningitis were never practiced by the physicians.

\section{Discussion}

Likewise, the global interest, research has been conducted at the local level in Pakistan. ${ }^{12-16}$ A study conducted in 2011 was conducted in different towns of Karachi, picking out general practitioners' knowledge and practices about dengue and malaria. Study findings revealed a high awareness of pathogenicity of dengue, types of stereotypes, dengue classification as a viral disease, vector born and blood-borne transmission. ${ }^{8}$ Similarly, in 2010 Syed M conducted a survey on knowledge, practices, and attitude among high and low socioeconomic adults group. According to the conducted study, an adequate knowledge was predicted among these group regarding Dengue process which includes transmission, symptoms, vector and etiology, Risk factors including time of day, seasons and locations, and Prevention strategies comprises of water storage, mats, and nets. ${ }^{9}$ In 2015, major cities of Pakistan which were being under attack of dengue were categorized for study populations by targeting physicians evaluate the knowledge and management practices of dengue. Results of this study declare most of the population of the physician were holding MBBS degree and their knowledge score were 62.5\%. Although no significant difference was predicted between knowledge score of physicians working in private or public healthcare setups. ${ }^{10}$ Likewise, these studies our study findings show most of the respondent belong to MBBS and have adequate knowledge about vector-borne, Aedes Mosquito, effective age group and stagnant water as a cause of dengue fever.

In those tropical and semi-urban countries, many studies enlighten the lack of knowledge which is responsible for the epidemiology of dengue in those areas. Another author conducted a survey of dengue knowledge, practices and attitude among healthcare practitioners in Southern Taiwan. The study indicated dengue was an endemic throughout in Taiwan with a scale of infection mostly corrected by respondent. ${ }^{11}$ Since Pakistan is an endemic country of dengue fever; ${ }^{12}$ data collection period was designed specially in dengue epidemic months. Not only that, data collection period was extended up to March, in which new house officer join for their training. Likewise, our study a study was conducted during the 2014 timeline by Dhimal et al in a high and lowland healthy population of central Nepal. ${ }^{17}$ Participants had easily identified the symptomatic manifestation of dengue fever whether they belong to low land or high land community of Nepal. Nevertheless, many respondents from low and high land were unaware of Aedes Mosquito as a vector of dengue and apart from it, every participant included in the study had the knowledge of blood-borne transmission of dengue fever. This study also enlightens the preventive measures which were taken by the community of high and low land, and almost every participant had the knowledge of preventive measures.

Additionally, Gunasekara et al conducted same KAP study in an urban community of Sri Lanka in 2012..$^{18}$ This Knowledge Attitude and Practices (KAP) study included questions about general medications which were used by the respondents; most of the respondent was unsure about the medical prescription. Moreover, only a few populations of respondents known use of Paracetamol and Aspirin medication accurate prescription. Overall Gunasekara et al, constructed result that half of the respondents have satisfactory knowledge about dengue symptomatic manifestation and most of the population had good practical knowledge. In 2014, a comprehensive research on urban, rural and semi-urban communities in Malaysia, ${ }^{19}$ according to this research there was no significant discrimination on socio-demographic and knowledge score between the communities of Malaysia. In the previous timeline i.e. 2010, two KAP studies regarding dengue infections at Westmoreland- Jamaica and Chennai city, Tamil Nadu-India were conducted. Both studies reflected the lack of knowledge about the dengue fever knowledge, behavior and practices, altho ${ }^{20,21}$ ugh physicians were not included in their study of our study respondents had blind knowledge about it. For this instance, 
a study conducted by Linda et al, extract the KAP study among the physicians of Singapore and likewise our study they include major proportion from the discipline of government subsidized polyclinics. ${ }^{22}$ This study claimed half of the population preferred dengue diagnostic tests frequently and used dengue serology tests ( $\operatorname{IgG} / \operatorname{IgM})$ as comparative purpose. Last but not least, Dengue Vaccine "Dengvaxia (CYD-TDV) by Sanofi Pasteur"23 were the best practice which had been evaluated during the study emphasizes the need of administration to all the strike communities and areas with a strong knowledge \& practice awareness among the physicians.

\section{Conclusion}

In spite of a major number of dengue patients in targeted cities, physicians have lack of knowledge about certain topics. Advance knowledge about dengue, symptomatic illness like pain behind the back of eyes is a symptom of dengue, vaccines of dengue, practices, and medication which should not be given to dengue fever or shock patients were found to be not known by the selected hospital physicians. It is recommended that awareness conferences symposiums and practices workshops must be scheduled in the future because it is the only way to minimize the proportion of higher dengue patients in Pakistan.

\section{Acknowledgements}

None.

\section{Conflict of interest}

The author declares there is no conflict of interest.

\section{References}

1. Guzman M, Halstead S, Artsob H, et al. Dengue a continuing global threat. Nat Rev Microbiol. 2010;8(12 Supple):S7-S16.

2. Chinnakali P, Gurnani N, Upadhyay R, et al. High level of awareness but poor practices regarding dengue fever control: A cross-sectional study from North India. N Am J Med Sci. 2012;4(6):278-282.

3. World Health Organization. WHO Executive Board requests resolution on a comprehensive approach to global vector control. 2017.

4. Barniol J, Gaczkowski R, Barbato E, et al. Usefulness and applicability of the revised dengue case classification by disease: multi-centre study in 18 countries. BMC Infectious Diseases. 2011;11:106.

5. Jahan F. Dengue Fever (DF) in Pakistan. Asia Pacific Family Medicine. 2011;10:1-4.

6. Mayxay M, Cui W, Thammavong S, et al. Dengue in peri-urban PakNgum district, Vientiane capital of Laos: a community survey on knowledge, attitudes and practices. BMC Public Health. 2013;13:434.
7. Zameer M, Shuja M, Ashraf A, et al. Knowledge attitudes and practices study of dengue viral infection and its association with environmental factors and health issues, Lahore Pakistan. AJEST. 2013;7:711-717.

8. Thaver A, Sobani Z, Qazi F, et al. Assessing the need for training: general practitioners' knowledge, attitude and practice concerning dengue and malaria in Karachi, Pakistan. Int Health. 2011;3(2):126-130.

9. Syed M, Saleem T, Syeda U, et al. Knowledge, attitudes and practices regarding dengue fever among adults of high and low socioeconomic groups. J Pak Med Assoc. 2010;60:243-7.

10. Rafique I, Saqib MAN, Siddiqui S, et al. Dengue knowledge and its management practices among physicians of major cities of Pakistan. JPMA. 2015;65:392-396.

11. Ho T, Huang M, Wang S, et al. Knowledge, attitude, and practice of dengue disease among healthcare professionals in southern Taiwan. $J$ Formos Med Assoc. 2013;112:18-23.

12. Koo C, Nasir A, Hapuarachchi H, et al. Evolution and heterogeneity of multiple serotypes of Dengue virus in Pakistan, 2006-2011. Virol J. $2013 ; 10: 275$.

13. WHO Report on Global Surveillance of Epidemic-prone Infectious Diseases - Dengue and dengue haemorrhagic fever? WHO. 2017.

14. Dengue and severe dengue. World Health Organization. 2017.

15. WHO EMRO. Dengue fever Programmes Pakistan. EMRO. WHO. 2018.

16. TDR. Handbook for clinical management of dengue. WHO. 2012.

17. Dhimal M, Aryal K, Dhimal M, et al. Knowledge, Attitude and Practice Regarding Dengue Fever among the Healthy Population of Highland and Lowland Communities in Central Nepal. PLoS ONE. 2014;9:e102028.

18. Gunasekara TDCP, Velathanthiri VGNS, Weerasekara MM, et al. Knowledge, attitudes and practices regarding dengue fever in a suburban community in Sri Lanka. Galle Medical Journal. 2012;17:1017 .

19. Al-Dubai SA, Ganasegeran K, Mohanad Rahman A, et al. Factors affecting dengue fever knowledge, attitudes and practices among selected urban, semi-urban and rural communities in Malaysia. Southeast Asian J Trop Med Public Health. 2013;44(1):37-49.

20. Shuaib F, Todd D, Campbell-Stennett D, et al. Knowledge, attitudes and practices regarding dengue infection in Westmoreland, Jamaica. West Indian Med J. 2010;59(2):139-146.

21. Ashok Kumar VA, Rajendran R, Manavalan R, et al. Studies on community knowledge and behavior following a dengue epidemic in Chennai city, Tamil Nadu, India. Tropical Biomedicine. 2010;27:330336 .

22. Lee LK, Thein TL, Kurukularatne C, et al. Dengue Knowledge, Attitudes, and Practices among Primary Care Physicians in Singapore. Ann Acad Med Singapore. 2011;40:533-8.

23. Dengue vaccine research. World Health Organization. 2017. 